\title{
Three-Dimensional Assembly Tolerance Analysis Based on the Jacobian-Torsor Statistical Model
}

\author{
Heping Peng ${ }^{1}$ and Wenlong Lu ${ }^{2}$ \\ ${ }^{1}$ School of Electromechanical \& Architectural Engineering, Jianghan University, Wuhan 430056, China \\ ${ }^{2}$ School of Mechanical Science \& Engineering, Huazhong University of Science and Technology, Wuhan 430074, China
}

\begin{abstract}
The unified Jacobian-Torsor model has been developed for deterministic (worst case) tolerance analysis. This paper presents a comprehensive model for performing statistical tolerance analysis by integrating the unified Jacobian-Torsor model and Monte Carlo simulation. In this model, an assembly is sub-divided into surfaces, the Small Displacements Torsor (SDT) parameters are used to express the relative position between any two surfaces of the assembly. Then, 3D dimension-chain can be created by using a surface graph of the assembly and the unified Jacobian-Torsor model is developed based on the effect of each functional element on the whole functional requirements of products. Finally, Monte Carlo simulation is implemented for the statistical tolerance analysis. A numerical example is given to demonstrate the capability of the proposed method in handling three-dimensional assembly tolerance analysis.
\end{abstract}

\section{Introduction}

With the release of new generation GPS tolerancing standards, geometric tolerances are generally accepted as industry practices [1]. Geometric tolerances and dimensional tolerances, as well as the interaction between them in the tolerance zone can be taken into consideration by $3 \mathrm{D}$ tolerance analysis methods. Desrochers et al. [2] proposed the unified Jacobian-Torsor model for deterministic tolerance analysis by the unifying of two existing models: the torsor model and the Jacobian matrix model in which the torsor model is used for tolerance representation and the Jacobian matrix for tolerance propagation. Currently, the research on combinations of 3D tolerance analysis technology and statistical analysis method is attracting the attention of many researchers [3, 4]. This paper focuses on the development of an analysis method to realize the statistical tolerance analysis of a mechanical assembly by integrating the unified Jacobian-Torsor model with Monte Carlo simulation.

\section{Unified Jacobian-Torsor model}

\subsection{Surface graph of an assembly}

The surface model concept is a basic concept within the new generation GPS standards system and is an abstract model of the physical interface between a workpiece and its environment [5]. Teissandier et al. [6] presented the method to describe an assembly using surface model. For example, the assembly shown in Fig.1 comprises seven parts, and the parts can be subdivided into ideal surfaces.
The designation of a surface is made up of two numbers separated by a ",": $(1,1)$ means surface 1 of part 1 .

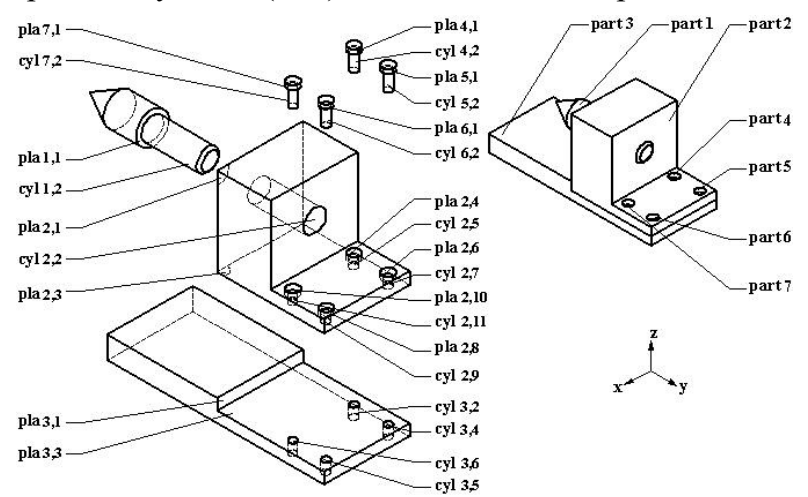

Figure 1. Sub-division of an assembly into surfaces

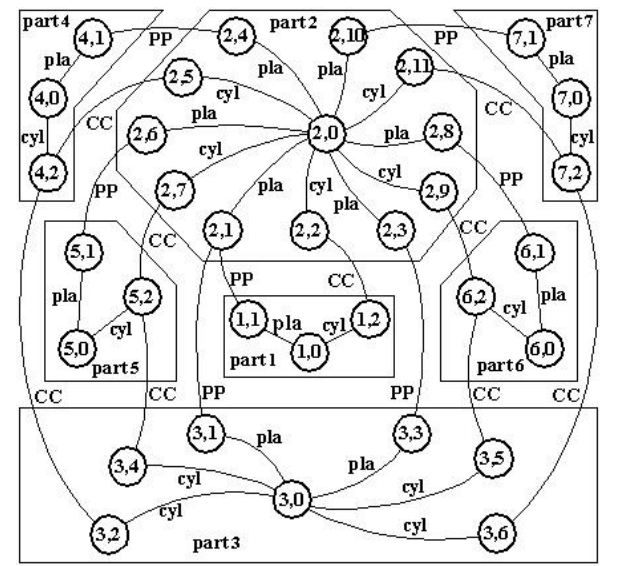

pla: tolerance zone of plane surface CC: joint between two coaxial cylinders cyl: tolerance zone of cylindrical surface PP: joint between two merged planes Figure 2. Surface graph of the assembly (Fig. 1) 
The part 1 (see Fig. 1) is divided into two surfaces: plane $(1,1)$ and cylinder $(1,2)$. The vertex $(1,0)$ corresponds to nominal surfaces of part 1. Any edge between vertices $(i, 0)$ and $(i, j)$ symbolizes the tolerance zone associated with surface $(i, j)$. Any edge between vertices $(i, j)$ and $(k, l)$ with $i \neq k ; j \neq 0$ and $l \neq 0$ symbolizes a joint (see Fig. 2). The surface graph of the assembly can be created as shown in Fig. 2.

\subsection{Creation of a 3D dimension-chain}

In assembly tolerance analysis, all variations should be quantitatively described including dimensional and geometric feature variations of each individual part in the assembly and kinematic variations occurring in the contacts or joints between mating parts. After an assembly is subdivided into surfaces, the relative position between any two surfaces of an assembly is determined

$$
\begin{aligned}
& {\left[\begin{array}{c}
{\left[\alpha^{-}, \alpha^{+}\right]} \\
{\left[\beta^{-}, \beta^{+}\right]} \\
{\left[\gamma^{-}, \gamma^{+}\right]} \\
{\left[u^{-}, u^{+}\right]} \\
{\left[v^{-}, v^{+}\right]} \\
{\left[w^{-}, w^{+}\right]}
\end{array}\right]_{F R}=\left[[ J _ { 1 } J _ { 2 } J _ { 3 } J _ { 4 } J _ { 5 } J _ { 6 } ] _ { F E 1 } \cdots [ J _ { 1 } J _ { 2 } J _ { 3 } J _ { 4 } J _ { 5 } J _ { 6 } ] _ { F E k } \cdots \left[J_{1}\right.\right.} \\
& {\left[\begin{array}{c}
{\left[\alpha^{-}, \alpha^{+}\right]} \\
{[\beta-, \beta+} \\
{\left[\gamma^{-}, \gamma^{+}\right]} \\
{\left[u^{-}, u^{+}\right]} \\
{\left[v^{-}, v^{+}\right]} \\
{\left[w^{-}, w^{+}\right]}
\end{array}\right]_{F R} \text { is SDT matrix associated to assembly }}
\end{aligned}
$$

functional requirement (FR); $\left[\begin{array}{c}{\left[\alpha^{-}, \alpha^{+}\right]} \\ {\left[\beta^{-}, \beta^{+}\right]} \\ {\left[\gamma^{-}, \gamma^{+}\right]} \\ {\left[u^{-}, u^{+}\right]} \\ {\left[v^{-}, v^{+}\right]} \\ {\left[w^{-}, w^{+}\right]}\end{array}\right]_{F E k}$ is SDT matrix

of the $k^{\text {th }}(k=1,2, \cdots, n)$ functional element pair; $n$ is the number of torsors in the tolerancing chain; $\alpha^{-}, \beta^{-}, \gamma^{-}, u^{-}, v^{-}$, $w^{-}$and $\alpha^{+}, \beta^{+}, \gamma^{+}, u^{+}, v^{+}, w^{+}$are lower and upper limits of

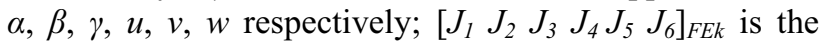
Jacobian matrix expressing a geometrical relation between the assembly functional requirement and the $k^{\text {th }}$ functional element pair, and $J_{1} J_{2} J_{3}$ are three rotation components; $J_{4} J_{5} J_{6}$ are three translation components; The multiplication operation in Eq.(1) uses the interval arithmetic [2].

The Jacobian matrix is formulated as:

$$
\left[J_{1} J_{2} \cdots J_{6}\right]_{F E \mathrm{k}}=\left[\begin{array}{ll}
{\left[R_{0}^{k}\right]_{3 \times 3} \cdot\left[R_{P T}\right]_{3 \times 3}} & {\left[W_{k}^{n}\right]_{3 \times 3} \cdot\left[R_{0}^{k}\right]_{3 \times 3} \cdot\left[R_{P T}\right]_{3 \times 3}} \\
{[0]_{3 \times 3}} & {\left[R_{0}^{k}\right]_{3 \times 3} \cdot\left[R_{P T}\right]_{3 \times 3}}
\end{array}\right]
$$

where $\left[R_{0}^{k}\right]_{3 \times 3}$ is the orientation matrix of reference frame $k$ relative to 0 , and reference frame 0 is the global reference frame; $\left[R_{P T}\right]_{3 \times 3}$ is the projection matrix whose purpose is to establish the projection of a traditional torsor along the direction of analysis of the tolerancing chain for the tilted tolerance zone; $\left[W_{k}^{n}\right]_{3 \times 3}$ is a Skew-symmetric with the aid of a set of SDT parameters. That is, it is possible to express the limits of relative small displacements between an associated surface and a nominal surface inside a tolerance zone, and the limits of relative displacements between two associated surfaces of two different parts in a joint. In order to determine the torsor between any two surfaces, two governing rules are defined: the union and the intersection. Based on the effect of each functional element on the whole functional requirements of products, a $3 \mathrm{D}$ dimension-chain can be created.

\subsection{Jacobian-Torsor model of tolerance analysis}

Based on the combination of the Jacobian model and the torsor based representation of tolerance zones, the unified Jacobian-Torsor model proposed by Desrochers et al [2] is represented as:

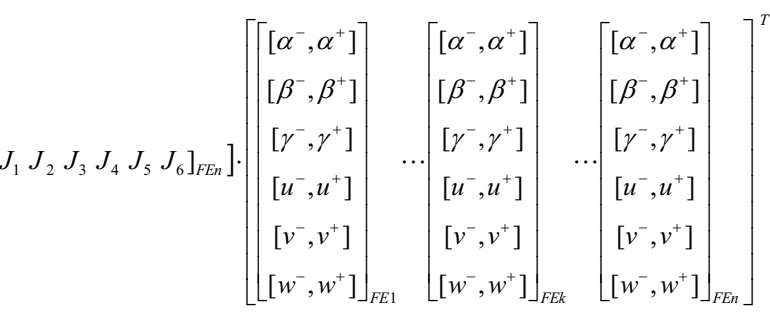

matrix allowing the representation of vector $\left[\overrightarrow{d_{n}}-\overrightarrow{d_{k}}\right]$ with $d x_{k}^{n}=d x_{n}-d x_{k}, d y_{k}^{n}=d y_{n}-d y_{k}, d z_{k}^{n}=d z_{n}-d z_{k}$, and $\vec{d}_{k}=\left[\begin{array}{lll}d x_{k} & d y_{k} d z_{k}\end{array}\right]^{T}$ is the position vector defining the position of the origin of reference frame $k$ in 0 . It can be defined as follows:

$$
\left[W_{k}^{n}\right]_{3 \times 3}=\left[\begin{array}{ccc}
0 & -d z_{k}^{n} & d y_{k}^{n} \\
d z_{k}^{n} & 0 & -d x_{k}^{n} \\
-d y_{k}^{n} & d x_{k}^{n} & 0
\end{array}\right]
$$

\section{Statistical tolerance analysis}

The aim of statistical tolerance analysis is to get the statistical limits of the assembly functional requirement $(F R)$ in the desired direction. Yet, the model in Eq. (1) is deterministic tolerance analysis model. In order to perform statistical tolerance analysis, a Monte Carlo simulation will be used to iteratively evaluate the deterministic model using sets of random numbers as inputs. Once after a great many of random numbers are used as inputs, the deterministic model will become a stochastic model.

It is well known that the functional requirements for a complex mechanical assembly are normally evaluated by several assembly critical dimensions. These critical dimensions are simultaneously controlled within certain variation ranges for the best working performances [7]. One of the assembly response functions is given by:

$$
z=g\left(y_{1}, y_{2}, \cdots, y_{\mathrm{n}}\right)
$$

where $y_{k}(k=1,2, \cdots, n)$ is component design dimension. Using Taylor series expansion and neglecting the 
higher-order terms beyond the first order, Eq. (2) can be approximated as:

$$
\Delta \mathrm{z}=\sum_{k=1}^{n}\left(\left.\frac{\partial g}{\partial y_{k}}\right|_{\bar{y}} \cdot \Delta y_{k}\right)
$$

where $\Delta z$ is the variation of assembly critical dimension. $\Delta y_{k}$ is the deviation of $y_{k}$ from its mean value $\overline{y_{k}}$, $\Delta y_{k}=y_{k}-\overline{y_{k}}$. In the bilateral tolerance system, the nominal value of the dimensions is just the mean value of the dimensions.

Statistical tolerance analysis makes use of the relationship expressed by Eq. (3), in which the random variables are assigned as input variables. Random number generators are used to generate a sample of numbers $\Delta y_{l}$, $\Delta y_{2}, \ldots, \Delta y_{n}$. The value of $\Delta z$ corresponding to this sample can be computed. This process is replicated a large number of samples, we will gain a random sample for $\Delta z$. The mean and standard deviation of the output $\Delta z$ (that is $F R$ ) can be determined through statistical analysis of this sample.

This process comprises the following steps:

- Creation of the unified Jacobian-Torsor model for the assembly tolerance analysis. According to Eq. (1), SDT matrixes of all functional element pairs and the corresponding Jacobian matrixes will be determined in this step.

- Generate real random values for each SDT component. Several software packages can generate random variables, such as we can use the function Rand ( ) in Microsoft Excel. It's important to note that the random values should be within the bounds of the intervals defined by each SDT.

- Compute real FR values for each set of random values. Simulation was stopped as the number of iterations increases past the limited number, and all these simulated data are recorded in a data structure of the program.

- Analyze the results and determine mean and standard deviation of $F R$ in the direction of tolerance analysis.

\section{Numerical example}

In this section, a numerical example is given to illustrate the application of the proposed tolerance analysis model. The assembly consists of two blocks with inclined surfaces (parts 1 and 2) and a space (part 3) as shown in Fig. 3. The functional requirement of this assembly is the contact gap, shown on the rightmost of the assembly, which is the critical characteristic of this assembly wish to control.

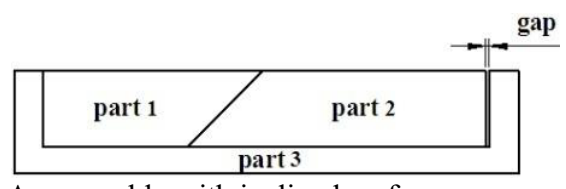

Figure 3. An assembly with inclined surfaces

The relevant dimensions and tolerances of the three parts are shown in Fig. 4.

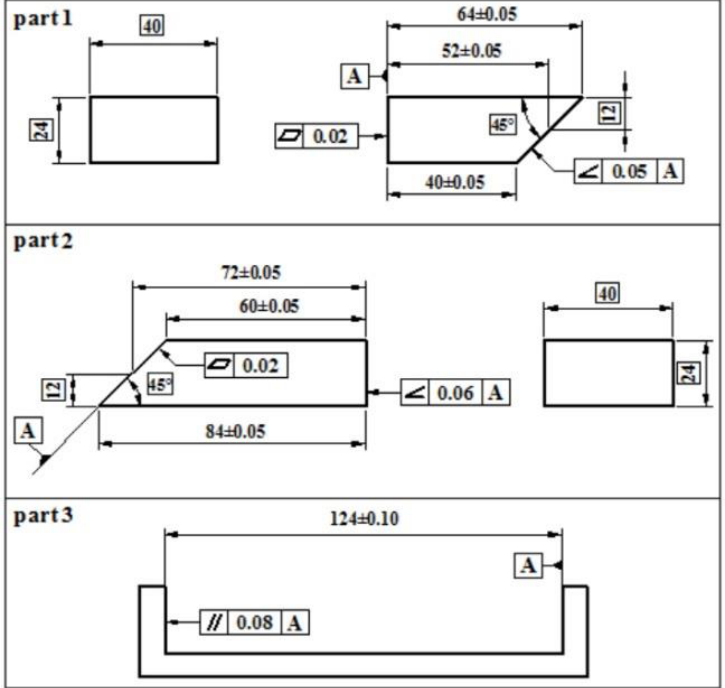

Figure 4. Detail drawing of part 1-3

In order to determine the tolerance value of the contact gap (Fig. 3), the effective feature surfaces between two parts are identified. There are six effective feature surfaces in this assembly as shown in Fig. 5. The designation of a surface is made up of two number separated by a "," e.g., $\mathrm{S}_{l, 1}$ means surface 1 of part 1 . The geometric variations of these features can be calculated based proposed modeling method at first, and then these variations can be accumulated to derive the required tolerance value.

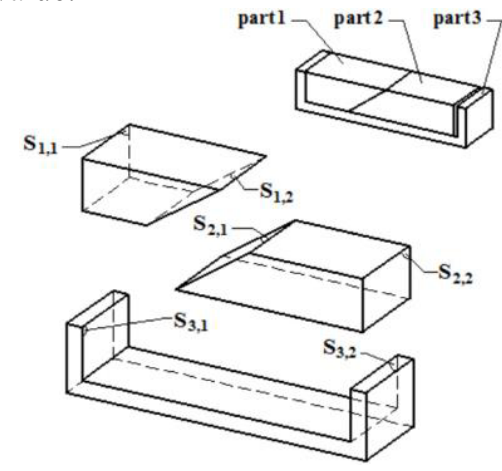

Figure 5. Effective features

The local coordinate systems are established on the effective features as shown in Fig. 6.

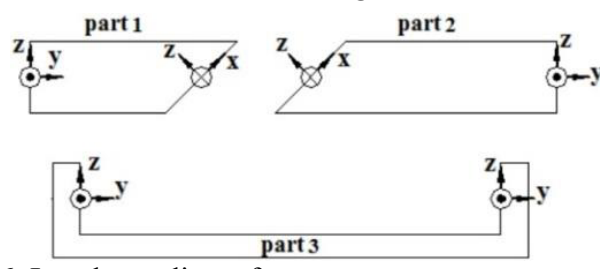

Figure 6. Local coordinate frames

After identifying all features and setting of local coordinate frames, the surface graph of the assembly can be constructed. Considering the Union rule of torsors, torsor $\boldsymbol{\tau}_{2,2 / 3,2}$ associated to the functional requirement consists of torsors $\boldsymbol{\tau}_{2,2 / 2,1}, \boldsymbol{\tau}_{2,1}, \boldsymbol{\tau}_{2,1 / 1,2}, \boldsymbol{\tau}_{1,2 / 1,1}, \boldsymbol{\tau}_{1,1}, \boldsymbol{\tau}_{1,1 / 3,1}$ and $\boldsymbol{\tau}_{3,1 / 3,2}$. The two kinematic torsors $\boldsymbol{\tau}_{2,1 / 1,2}$ and $\boldsymbol{\tau}_{1,1 / 3,1}$ are considered null because the contact between the two planes is assumed perfect. 
According to Eq. (1), we can obtain a unified Jacobian-Torsor model about the FR in this assembly which can be written as follows, where the variables $\mathrm{C}$ and $\mathrm{S}$ correspond respectively to $\cos 45^{\circ}$ and $\sin 45^{\circ}$.

$\left[\begin{array}{c}{\left[\alpha^{-}, \alpha^{+}\right]} \\ {\left[\beta^{-}, \beta^{+}\right]} \\ {\left[\gamma^{-}, \gamma^{+}\right]} \\ {\left[u^{-}, u^{+}\right]} \\ {\left[v^{-}, v^{+}\right]} \\ {\left[w^{-}, w^{+}\right]}\end{array}\right]_{F R}$

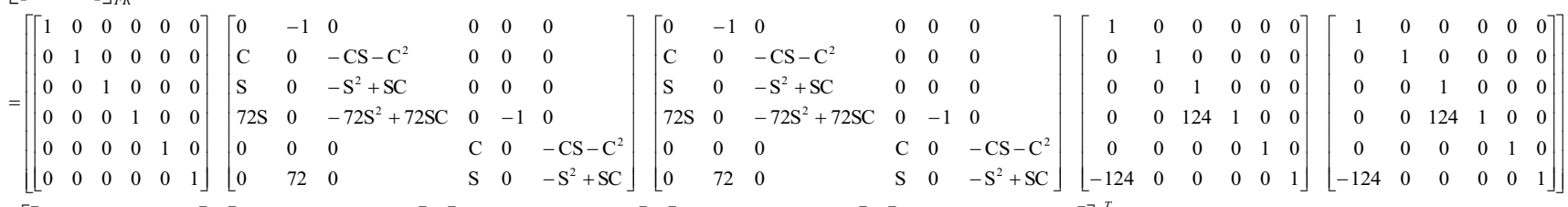
• $\left[\left[\begin{array}{c}{[-0.0025,+0.0025]} \\ {[0,0]} \\ {[-0.0015,+0.0015} \\ {[0,0]} \\ {[-0.05,+0.05]} \\ {[0,0]}\end{array}\right]\left[\begin{array}{c}{[-0.00050,+0.00050]} \\ {[-0.00059,+0.00059]} \\ {[0,0]} \\ {[-0.01,+0.01]} \\ {[0,0]} \\ {[-0.01414,+0.01414]}\end{array}\right]\left[\begin{array}{c}{[-0.00125,+0.00125]} \\ {[-0.00147,+0.00147]} \\ {[0,0]} \\ {[-0.03536,+0.03536]} \\ {[0,0]} \\ {[-0.05,+0.05]}\end{array}\right]\left[\begin{array}{c}{[-0.00083,+0.00083]} \\ {[0,0]} \\ {[-0.00050,+0.00050]} \\ {[0,0]} \\ {[-0.01,+0.01]} \\ {[0,0]}\end{array}\right]\left[\begin{array}{c}{[-0.00333,+0.00333]} \\ {[0,0]} \\ {[-0.002,+0.002]} \\ {[0,0]} \\ {[-0.10,+0.10]} \\ {[0,0]}\end{array}\right]\right]^{T}$

Using the interval algorithm, we draw the conclusion that the functional requirement or contact gap of this assembly in the $y$ direction must lie in an interval of $[-0.25621,+0.25621]$.

In the statistical approach, statistical torsor parameters of functional element pairs are listed in Table 1.

Table 1. Statistical torsor parameters of functional element pairs

\begin{tabular}{|c|c|c|c|}
\hline Torsors & Components of Torsors & $\begin{array}{c}\text { Specified } \\
\text { distributio } \\
\mathrm{n} / \\
\text { Rejected } \\
\end{array}$ & $\begin{array}{l}\text { Statistical } \\
\text { parameters }\end{array}$ \\
\hline$\tau_{2,2 / 2,1}$ & $\begin{array}{l}\left\{\begin{array}{c}{\left[\alpha^{-}, \alpha^{+}\right]} \\
{\left[\beta^{-}, \beta^{+}\right]} \\
{\left[\gamma^{-}, \gamma^{+}\right]}\end{array}\right\}=\left\{\begin{array}{c}{[-0.0025,+0.0025]} \\
{[0,0]} \\
{[-0.0015,+0.0015]}\end{array}\right\} \\
\left\{\begin{array}{c}{\left[u^{-}, u^{+}\right]} \\
{\left[v^{-}, v^{+}\right]} \\
{\left[w^{-}, w^{+}\right]}\end{array}\right\}=\left\{\begin{array}{c}{[0,0]} \\
{[-0.05,+0.05]} \\
{[0,0]}\end{array}\right\}\end{array}$ & $\begin{array}{c}\text { Normal/ } \\
5 \%\end{array}$ & $\begin{array}{l}\mu=0.00, \sigma=0.00128 \\
\mu=0.00, \sigma=0.00 \\
\mu=0.00, \sigma=0.00077 \\
\mu=0.00, \sigma=0.00 \\
\mu=0.00, \sigma=0.02551 \\
\mu=0.00, \sigma=0.00\end{array}$ \\
\hline$\tau_{2,1}$ & 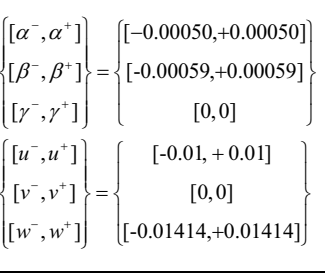 & $\begin{array}{c}\text { Normal/ } \\
5 \%\end{array}$ & $\begin{array}{l}\mu=0.00, \sigma=0.00026 \\
\mu=0.00, \sigma=0.00030 \\
\mu=0.00, \sigma=0.00 \\
\mu=0.00, \sigma=0.00510 \\
\mu=0.00, \sigma=0.00 \\
\mu=0.00, \sigma=0.00721\end{array}$ \\
\hline$\tau_{1,2 / 1,1}$ & 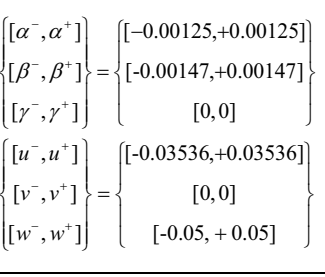 & $\begin{array}{c}\text { Normal/ } \\
5 \%\end{array}$ & $\begin{array}{l}\mu=0.00, \sigma=0.00064 \\
\mu=0.00, \sigma=0.00075 \\
\mu=0.00, \sigma=0.00 \\
\mu=0.00, \sigma=0.01804 \\
\mu=0.00, \sigma=0.00 \\
\mu=0.00, \sigma=0.02551\end{array}$ \\
\hline$\tau_{1,1}$ & $\begin{array}{l}\left\{\begin{array}{l}{\left[\alpha^{-}, \alpha^{+}\right]} \\
{\left[\beta^{-}, \beta^{+}\right]} \\
{\left[\gamma^{-}, \gamma^{+}\right]}\end{array}\right\}=\left\{\begin{array}{c}{[-0.00083,+0.00083]} \\
{[0,0]} \\
{[-0.00050,+0.00050]}\end{array}\right\} \\
\left\{\begin{array}{c}{\left[u^{-}, u^{+}\right]} \\
{\left[v^{-}, v^{+}\right]} \\
{\left[w^{-}, w^{+}\right]}\end{array}\right\}=\left\{\begin{array}{c}{[0,0]} \\
{[-0.01,+0.01]} \\
{[0,0]}\end{array}\right\}\end{array}$ & $\begin{array}{c}\text { Normal/ } \\
5 \%\end{array}$ & $\begin{array}{l}\mu=0.00, \sigma=0.00042 \\
\mu=0.00, \sigma=0.00 \\
\mu=0.00, \sigma=0.00026 \\
\mu=0.00, \sigma=0.00 \\
\mu=0.00, \sigma=0.00510 \\
\mu=0.00, \sigma=0.00\end{array}$ \\
\hline
\end{tabular}

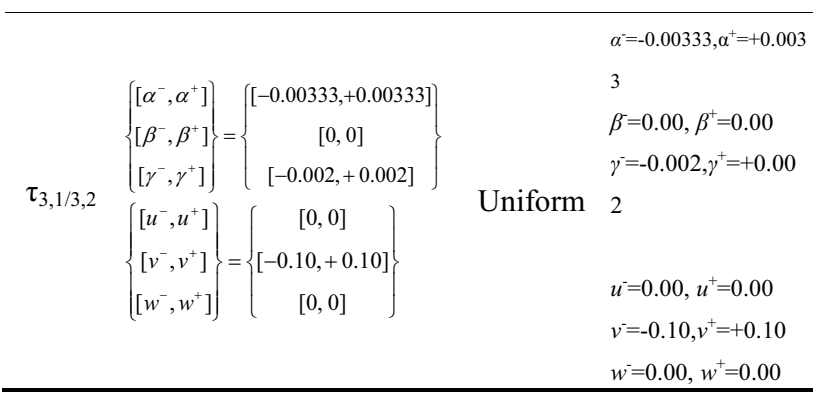

Simulation was stopped after 1000 iterations (can obtain 1000 values for each of the six torsor component values for FR). According to the functional requirement of this assembly, it is necessary to perform a statistical analysis for the $\mathrm{v}$ torsor component values for FR. The histogram of FR along the direction of analysis is shown in Fig. 7; the figure shows the FR data distribution is normal distribution. Thus, the functional requirement of this assembly in the direction of analysis determined by statistical analysis is: $[-0.13880,+0.13880]$, the corresponding rejection percentage is $5 \%$.

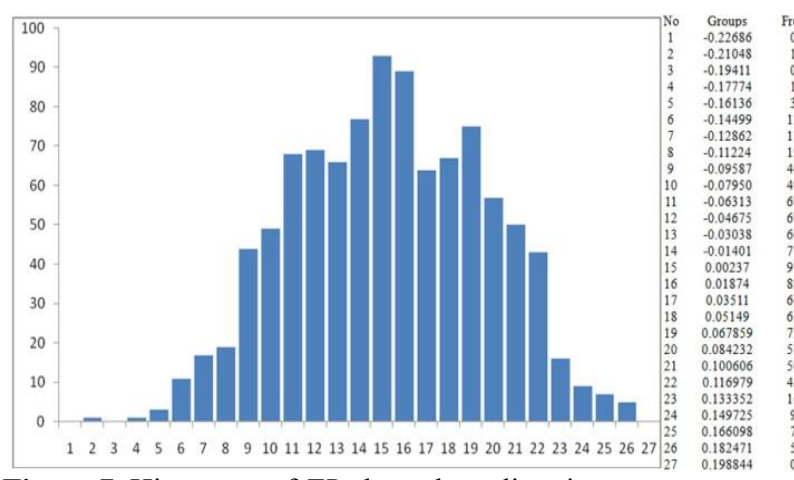

Figure 7. Histogram of $F R$ along the $y$ direction

\section{Conclusions}

The assembly tolerance analysis provides an effective evaluation tool for estimating the effects of variations caused by the dimensional and geometrical feature 
variations of the parts in an assembly on assembly functional requirement. This paper has described an analytical model integrated with the Monte Carlo simulation tool to obtain an algorithm which performs the statistical tolerance analysis of a mechanical assembly. The proposed method can be easily implemented in CAT systems and will provide a more effective manner for design and manufacturing engineers to deal with the statistical tolerance analysis.

\section{Acknowledgment}

The authors would like to acknowledge the financial support of the National Natural Science Foundation of the People's Republic of China (No. 51575235).

\section{References}

1. ISO 1101: Geometric Product Specifications (GPS)-Geometrical tolerancing-tolerancing of form, orientation, location and run-out, (2004)

2. A. Desrochers, W. Ghie and L. Laperrière, J. Comput. Inf. Sci. Eng. 3, 1 (2003)

3. W. Ghie, Int. J. Multiphys. 3, 1 (2009)

4. W. Ghie, L. Laperrière and A. Desrochers, Int. J. Prod. Res. 48, 15 (2010)

5. F. Charpentier, A. Ballu and J. Pailhès, Proceedings of the 12th CIRP conference on computer aided design, (2012).

6. D. Teissandier, Y. Couétard and A. Gérard, Comput. Aided Des., 31 (1999)

7. H. P. Peng, X. Q. Jiang and X. J. Liu, Int. J. Prod. Res. 46, 24 (2008) 